\title{
The Existence of Symptomatic Localized Lesion(s) and $\leq 4$ Lesions Are Indications for Radiotherapy: Screening Computed Tomography Images of Patients with Metastatic/Recurrent/Inoperable Cancer in a Single Japanese Institution Observational Study
}

\author{
Shinsaku Yamaguchi ${ }^{*}$, Yuichi Matsuki', Shinji Yotsumoto', Hiroaki Satou ${ }^{2}$, \\ Michiko Watanabe ${ }^{1}$, Sachie Yotsumoto ${ }^{1}$ \\ ${ }^{1}$ Department of Radiology, Kitakyushu General Hospital, Kitakyushu, Japan \\ ${ }^{2}$ Department of Forensic Medicine, School of Medicine, University of Occupational and Environmental Health, \\ Kitakyushu, Japan \\ Email: "specialvirtue38@yahoo.co.jp, s.yotsumoto@gmail.com, sato-hiroaki@aist.go.jp, \\ piyopiyo0818@hotmail.com, sachieyt@gmail.com,h-ooki@med.uoeh-u.ac.jp, s-ide@med.uoeh-u.ac.jp
}

Received 30 January 2016; accepted 26 February 2016; published 29 February 2016

Copyright (C) 2016 by authors and Scientific Research Publishing Inc.

This work is licensed under the Creative Commons Attribution International License (CC BY).

http://creativecommons.org/licenses/by/4.0/

(c) () Open Access

\footnotetext{
Abstract

Background: To investigate the prevalence of indications for radiotherapy (RT) in patients with metastatic/recurrent/inoperable cancer. We also sought to analyze such patients' clinical and radiological characteristics for indications of radiotherapy in those patients who had either surveillance or an initial assessment done by computed tomography (CT). Methods: Two diagnostic radiologists and a radiation oncologist evaluated a total of 13,225 consecutive patients from January 2012 to December 2012 at a single Japanese institution. Patients with metastatic/recurrent/ inoperable cancer were selected for further study. After two diagnostic radiologists identified patients with a detectable cancerous lesion, a radiation oncologist subsequently investigated whether there was any indication for RT. The oncologist also evaluated the relationship between patients' clinical/radiological factors, and patients with or without indications for RT. Results: Two diagnostic radiologists selected 329 patients showing a detectable gross cancerous lesion. In this

${ }^{*}$ Corresponding author.

How to cite this paper: Yamaguchi, S., et al. (2016) The Existence of Symptomatic Localized Lesion(s) and $\leq 4$ Lesions Are Indications for Radiotherapy: Screening Computed Tomography Images of Patients with Metastatic/ Recurrent/Inoperable Cancer in a Single Japanese Institution Observational Study. International Journal of Medical Physics, Clinical Engineering and Radiation Oncology, 5, 110-119. http://dx.doi.org/10.4236/ijmpcero.2016.51012
} 
patient group, a radiation oncologist identified 196 patients with metastatic/recurrent/inoperable cancer, of which 96 patients (49\%) showed an indication for RT. According to both univariate and multivariate analyses, $\leq 4$ lesions were significantly associated with patients who showed an indication for RT ( $P=0.0002$ and $P<0.0001$, respectively). The existence of symptomatic, localized lesion(s) was also significantly associated with those who showed an indication for RT (P < 0.001 and $P>0.001$, respectively). Conclusions: In screening CT images, approximately half of all patients with metastatic/recurrent/inoperable cancer showed an indication for RT. Moreover, $\leq 4$ lesions and/or the existence of a symptomatic, localized lesion were highly suggestive of an indication for RT. These findings would be of considerable interest to radiation oncologists planning appropriate treatments for cancer patients.

\section{Keywords}

Surveillance CT, Radiation Oncologist, Indication for Radiotherapy

\section{Introduction}

Radiotherapy (RT) is an essential and well-established treatment, used in the management of many types of malignancies, and plays a major role in a curative, adjuvant setting, as well as in palliation [1]-[5]. Previous reports suggest that $50 \%-60 \%$ of all cancer patients could potentially benefit from RT [4] [6]-[8]. However, in Japan, RT is used in less than $30 \%$ of cancer patients [9]. Such an obvious underutilization of RT is undesirable not only because it offers potentially curative treatment, but also because of its potential for treatment with a palliative intent. Since a lack of knowledge concerning indications for RT may be a crucial reason for its underuse, we thus decided to investigate RT practice at Kitakyushu General Hospital.

In a cancer management setting, computed tomography (CT) scans are increasingly being performed. Improvements in tumor evaluation by diverse imaging modalities, such as ultrasonography, magnetic resonance imaging (MRI), and fluorine-18-labeled fluoro-2-deoxy-D-glucose positron emission tomography (FDG-PET), have significantly improved both treatment decisions and the accuracy of highly precise RT. However, CT is still very important because of its easy interpretation and convenience. For patients with metastatic/recurrent cancer according to the Response Evaluation Criteria in Solid Tumors (RECIST) [10] [11], assessing the antitumor effects of systemic therapy using an image modality (mainly CT) is important in order to estimate the efficacy of therapies, and is also a key to guide potential changes in treatment.

However, because many non-radiation oncologists, including diagnostic radiologists, do not have adequate knowledge concerning the appropriate indications for RT in a variety of situations, the evaluation of CT scans for patients with metastatic/recurrent/inoperable cancer would not be very useful. In fact, we have noted many patients who missed the timing of radical, semi-radical, and palliative RT because of delays in consultations. RT referrals would logically correlate more closely with non-radiation oncologists' knowledge concerning the indications for RT. Therefore, as two diagnostic radiologists, together with a radiation oncologist, we screened CT images of patients with metastatic/recurrent/inoperable cancer, and assessed their radiological findings.

To our knowledge, there have not been any previous clinical reports concerning the analysis of CT images of patients with metastatic/recurrent/inoperable cancer by both diagnostic radiologists and radiation oncologists. Therefore, the purpose of this study was to investigate the prevalence of indications for RT in patients with metastatic/recurrent/inoperable cancer, who underwent either surveillance or an initial assessment by CT, and to evaluate the relationship between clinical/radiological factors and patients with or without indications for RT.

\section{Materials and Methods}

This study was approved by our institutional review board (Permission number: H25-11); informed consent from the subjects was waived for publication of this report and accompanying images.

\subsection{Selection of Patients}

We undertook a search of the radiology database (CT files) at our institution from January 2012 to December 
2012 and selected data from a total of 13,225 patients who underwent CT study. From among these patients, we selected a few for evaluation on the basis of the following strategies:

Firstly, a search in reports of interpretation and letters of request for CT scans was performed using any one of the following key words and Medical Subject Heading terms: "cancer", "tumor", and "malignant".

Secondly, two diagnostic radiologists (Y.M. and H.O.), working together, screened all CT images and further selected patients who had a cancerous lesion detectable by CT.

Finally, a radiation oncologist (S.Y.) diagnosed the latter patients with metastatic/recurrent/inoperable cancer, with reference to electronic medical records (including a complete history, physical examination by attending physician, laboratory data, tumor markers, and the patients' complaint).

\subsection{CT Image and Interpretation}

All CT images were obtained using a 16-MDCT scanner (Aquilion 16; Toshiba Medical Systems, Nasu, Japan). CT parameters were as follows: for chest CT $(0.5$-second rotation time; $120 \mathrm{kV} ; 150 \mathrm{~mA} ; 1.0-\mathrm{mm}$ beam collimation; beam pitch, 0.94 and 7.0-mm image slice thickness) and for abdominal CT (0.5-second rotation time; $120 \mathrm{kV} ; 300 \mathrm{~mA} ; 2.0-\mathrm{mm}$ beam collimation; beam pitch, 0.94 and 7.0-mm image slice thickness).

For 107 (55\%) out of 196 subjects, a contrast-enhanced CT image was available. For contrast-enhanced CT images, $100 \mathrm{~mL}$ of iohexol (300 mg I/mL; Omnipaque 300; Daiichi-Sankyo Co., Ltd., Tokyo, Japan) was injected intravenously at a rate of $1-1.5 \mathrm{~mL} / \mathrm{s}$, with a scan delay of $65-100$ seconds.

All CT images were interpreted jointly in agreement with two Japanese certificated diagnostic radiologists (Y.M. and H.O., with 17 and 10 years of experience, respectively).

\subsection{Indications for Radiotherapy}

A Japanese certificated radiation oncologist (S.Y., 10 years of experience) interpreted all CT images of patients selected by two diagnostic radiologists, and determined whether there were any indications for RT in those finally selected patients who had metastatic/recurrent/inoperable cancer. He divided them into four potential treatment intent groups: radical, semi-radical, palliative, and none, with reference to images by CT or other available modalities (ultrasonography, MRI, bone scintigraphy, and FDG-PET), as well as by electronic medical records. Radical intent pertained to cases at the CT scanning stage that had a good chance of being cured with RT as well as to cases for which standard initial treatment was performed. Semi-radical intent referred to cases at the CT scanning stage that had some chance of being cured by RT. Palliative intent referred to cases at the time of CT scanning, which had little chance of being cured by RT but were likely to get symptomatic relief. The remaining were cases at the CT scanning stage for which RT would not be beneficial due to each patient's clinical status.

\subsection{Statistical Analysis}

The relationships between clinical/radiological factors and patients with or without indications for RT were investigated using a Chi-square test, and with consideration of such factors as: age, gender, performance status (PS), cancer status, number of lesions, maximal tumor diameter, site of maximal tumor, symptomatic localized lesion, and primary site. Multivariate logistic regression analyses were performed to evaluate the association between clinical/radiological factors and patients with or without indications for RT.

\section{Results}

On the basis of the keywords ("cancer", "tumor", and "malignant"), we selected a total of 3456 patients for further study. Two diagnostic radiologists screened all CT images of such patients and further selected 329 patients who had a detectable gross cancerous lesion. Finally, a radiation oncologist identified 196 metastatic/recurrent/ inoperable cancer patients who met our inclusion criteria (Figure 1). Baseline clinical characteristics of patients are listed in Table 1. In this study, more than eighty percent of patients $(n=161,82 \%)$ were PS 2 or more. The major primary site for cancer was the colorectum (25\%), followed by the lung $(19 \%)$, stomach $(9 \%)$, or pancreas (9\%). Patients with metastatic cancer comprised more than half of all patients $(n=111,56 \%)$.

Table 2 outlines the results of a radiation oncologist's review which determined that $96(49 \%)$ of 196 patients with metastatic/recurrent/inoperable cancer showed indications for RT, the decision for which was made at his discretion. The RT intents of radical, semi-radical, and palliative treatment groups were $15(8 \%), 18(9 \%)$, and 


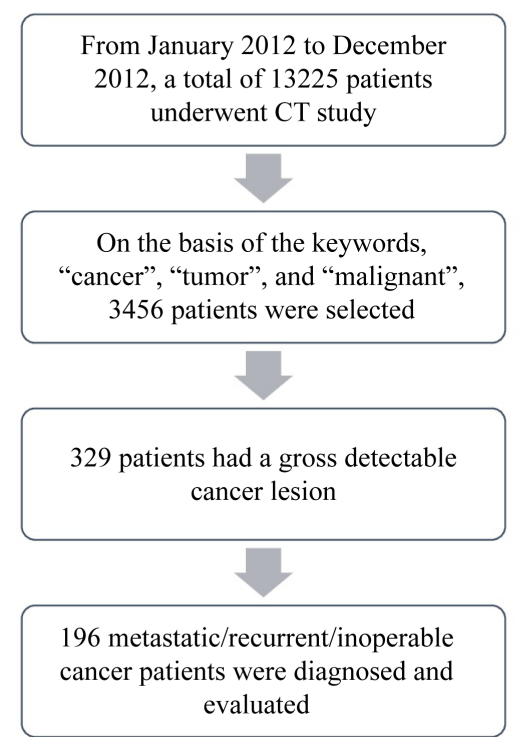

The remaining 3127 patients had neither a detectable cancerous lesion nor evidence of a recurrent cancer

The remaining 133 patients were non-metastatic and/or operable cancer patients

Figure 1. Profile of the patient selection procedure.

63 (32\%), respectively. The remaining 100 patients (51\%) showed no indications for RT (the none group) because a clinical benefit from RT was not expected.

The final treatments actually performed for the 196 patients, including surgery, chemotherapy, RT, hormonal therapy, or other supportive care only, were $13 \%(n=25), 25 \%(n=49), 18 \%(n=35), 4 \%(n=8)$, and $40 \%(n$ $=79$ ), respectively; supportive care without RT was the most applied treatment for metastatic/recurrent/inoperable cancer patients. In cases of applied RT, the median interval between CT evaluation and RT was 1.2 months (range $0.0-8.4$ ).

Figure 2 outlines the detailed treatments that were actually performed for 96 patients who showed an indication for RT: RT was the most common treatment $(35 / 96,37 \%)$, but surgery was also applied as a local therapy (12/96, 13\%), similar to RT. However, supportive care without RT (31/96, 32\%) was the second most common treatment, even though all 96 patients showed indications for RT. Figure 3 shows CT images of typical cases which were not referred for RT, in spite of having indications for RT.

The relationship between clinical/radiological factors and patients, with or without an indication for RT, is shown in Table 3. In univariate analyses using the Chi-square test, factors such as cancer status, number of lesions, site of maximal tumor, and the existence of symptomatic, localized lesions were significantly associated with patients who showed an indication for $\mathrm{RT}(\mathrm{P}=0.03, \mathrm{P}=0.0002, \mathrm{P}=0.05$, and $\mathrm{P}<0.0001$, respectively). In multivariate analyses of these significant factors using logistic regression, the number of lesions and the existence of a symptomatic, localized lesion were very significant $(\mathrm{P}<0.0001)$.

\section{Discussion}

To our knowledge, there are no previous clinical reports concerning the use of CT image interpretation, by both diagnostic radiologists and radiation oncologists, in investigations of the prevalence of indications for RT, and in evaluations of the relationship between clinical/radiological factors and patients with or without indications for RT. In our observational study, although 49\% (96/196) of metastatic/recurrent/inoperable cancer patients could have benefitted from RT, only $18 \%(35 / 196)$ were referred to a department of radiation oncology. In short, more patients could have been referred to receive RT, but instead low referral rates were noted for our institution.

A lack of referrals for RT could have been a consequence of a variety of reasons such as long wait times, limited consultation days, concerns about complications, uncertainty about the benefits, the functional status of a patient, and also patient preference; however, an important issue for our institution may have been a lack of understanding of RT on the part of physicians. For instance, Samant et al. demonstrated a direct relationship between the level of knowledge of RT and referral patterns in Ontario, Canada [12]. In this study, questionnaires were used to evaluate radiotherapy knowledge and mailed to a random sample of 400 practicing family physi- 
Table 1. Baseline clinical characteristics of 196 patients.

\begin{tabular}{|c|c|}
\hline Variable & n $(\%)$ \\
\hline \multicolumn{2}{|l|}{ Age (y) } \\
\hline Median (range) & $75(31-97)$ \\
\hline \multicolumn{2}{|l|}{ Gender } \\
\hline Male & $106(54)$ \\
\hline Female & $90(46)$ \\
\hline \multicolumn{2}{|l|}{ PS } \\
\hline $0 / 1 / 2 / 3 / 4$ & $3 / 32 / 81 / 62 / 18$ \\
\hline \multicolumn{2}{|l|}{ Primary site } \\
\hline Colorectum & $49(25)$ \\
\hline Lung & $37(19)$ \\
\hline Stomach & $18(9)$ \\
\hline Pancreas & $17(9)$ \\
\hline Liver & $14(7)$ \\
\hline Breast & $13(7)$ \\
\hline Kidney & $8(4)$ \\
\hline Bile duct & $7(4)$ \\
\hline Prostate & $6(3)$ \\
\hline Esophagus & $5(3)$ \\
\hline Other & $22(11)$ \\
\hline \multicolumn{2}{|l|}{ Cancer status } \\
\hline Metastatic & $111(56)$ \\
\hline Recurrence & $43(22)$ \\
\hline Inoperable & $42(21)$ \\
\hline \multicolumn{2}{|l|}{ No. of lesions } \\
\hline $0-4$ & $65(33)$ \\
\hline$\geq 5$ & $131(67)$ \\
\hline \multicolumn{2}{|l|}{ Histology } \\
\hline Adenocarcinoma & $103(53)$ \\
\hline Squamous cell carcinoma & $14(7)$ \\
\hline Hepatocellular carcinoma & $12(6)$ \\
\hline Small cell carcinoma & $5(3)$ \\
\hline Renal cell carcinoma & $5(3)$ \\
\hline Transitional cell carcinoma & $4(2)$ \\
\hline Other & $9(5)$ \\
\hline Unknown pathology & $44(22)$ \\
\hline
\end{tabular}

PS: Performance status. 
Table 2. A radiation oncologist's review.

\begin{tabular}{ll}
\hline Variable & $\mathrm{n}(\%)$ \\
\hline Indication for Radiotherapy $(\mathrm{n}=196)$ & $96(49)$ \\
Yes & \\
\hline Radiotherapy intent & $15(8)$ \\
Radical & $18(9)$ \\
Semi-radical & $63(32)$ \\
Palliative & $100(51)$ \\
No & \\
\hline Reason for Radiotherapy indication $(\mathrm{n}=96)$ & $37(39)$ \\
Pain & $30(31)$ \\
Possibility of a cure & $8(8)$ \\
Bleeding & $6(6)$ \\
Cosmetic problem & $5(5)$ \\
Dysphagia & $4(4)$ \\
Bowel obstruction & $2(2)$ \\
Brain metastases & $2(2)$ \\
Dyspnea & $2(2)$ \\
Other & \\
\hline
\end{tabular}

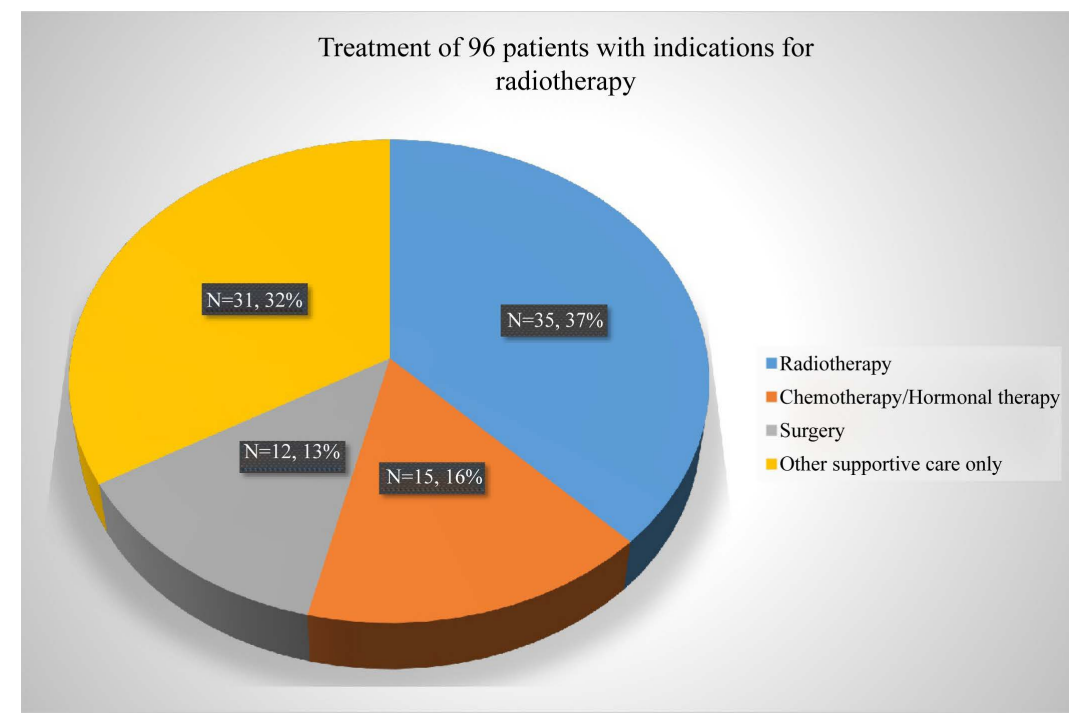

Figure 2. Treatments of 96 patients with indications for radiotherapy.

cians. A total of 172 completed surveys were analyzed, at an eligible response rate of $43 \%$ of surveyed physicians, $80 \%$ of whom were involved in providing palliative care for their patients. The authors reported that physicians who were more knowledgeable in regards to the common indications for palliative radiotherapy were significantly more likely to refer patients for radiotherapy $(\mathrm{P}<0.01)$. The authors concluded that many family physicians that have a less than optimal knowledge of the indications for palliative radiotherapy and the effectiveness of palliative radiotherapy, appeared less likely to refer cancer patients for radiotherapy. Similarly, Vulto 

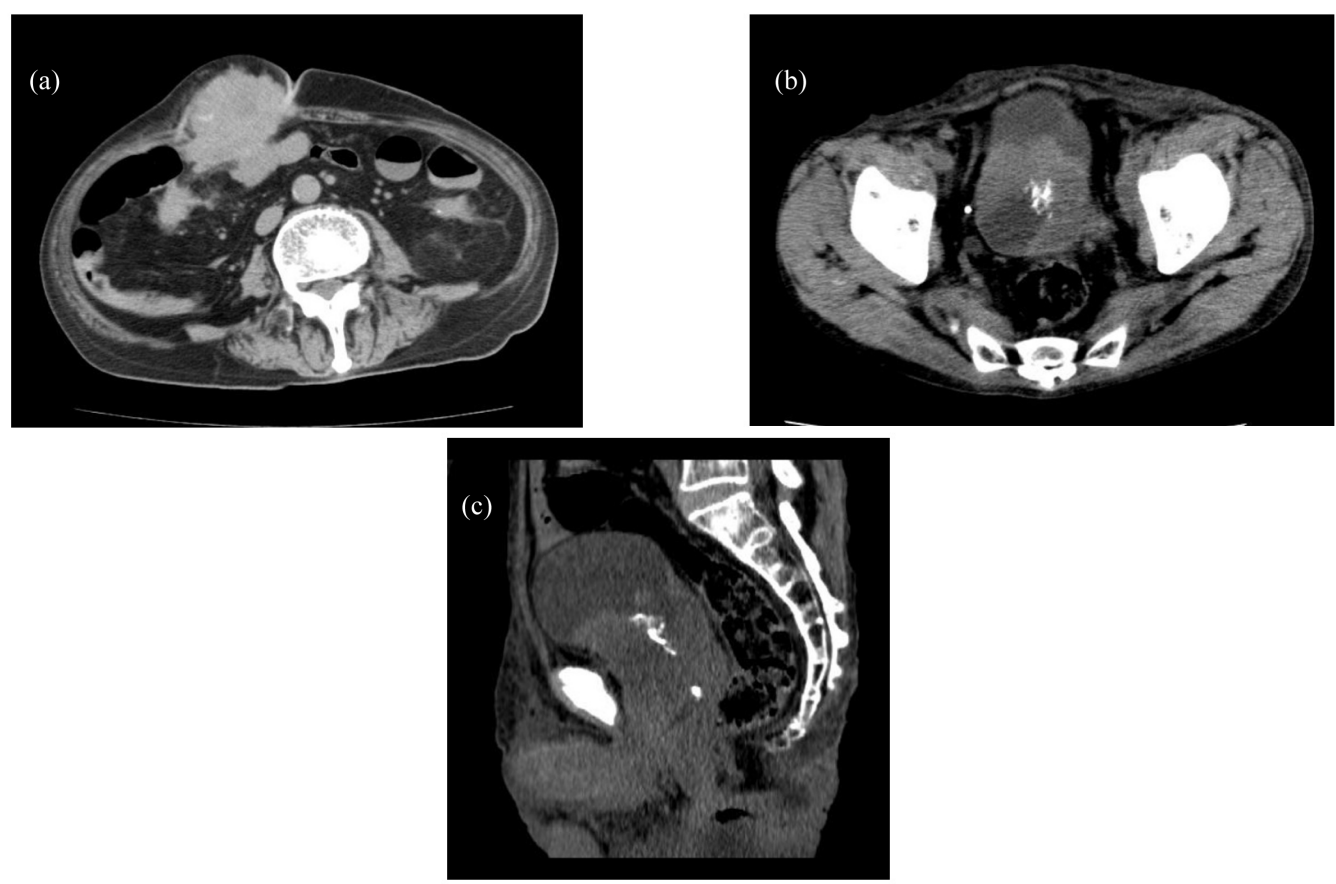

Figure 3. (a) An 85-year-old man with metastatic gastric cancer complained about both a cosmetic problem and pain caused by a metastatic tumor of the abdominal wall. This symptomatic, localized tumor was a good indication for palliative RT, but his symptoms were treated only by supported care. (b) (c) A 78year-old man with inoperable, advanced bladder cancer had hematuria. The primary tumor was an indication for radical RT, but his cancer was treated only by supported care.

Table 3. Relationship between clinical/radiological factors and patients, with or without RT indication, by Chi-square test.

\begin{tabular}{lcccc}
\hline \multirow{2}{*}{ Clinical factor } & \multicolumn{2}{c}{ Indication for Radiotherapy } & univariate & multivariate \\
\cline { 2 - 5 } & Yes $(\mathrm{n}=96)$ & No $(\mathrm{n}=100)$ & $\mathrm{P}$ & $\mathrm{P}$ \\
\hline Age $(\leq 75 />75)$ & $46 / 50$ & $47 / 53$ & 0.90 & - \\
Gender (male/female) & $54 / 42$ & $52 / 48$ & 0.55 & - \\
PS ( $\leq 2 />2)$ & $55 / 41$ & $61 / 39$ & 0.60 & - \\
Cancer status (Metastatic/Recurrence/Inoperable) & $46 / 22 / 28$ & $65 / 21 / 14$ & 0.03 & 0.90 \\
No. of lesions (0 - 4/ $\geq 5)$ & $44 / 52$ & $21 / 79$ & 0.0002 & $<0.0001$ \\
Maximal tumor diameter $(0-6 \mathrm{~cm} / \geq 6 \mathrm{~cm})$ & $54 / 42$ & $66 / 34$ & 0.16 & - \\
Site of maximal tumor (Chest/Abdomen/Bone and soft tissue/Other) & $23 / 50 / 18 / 5$ & $26 / 62 / 6 / 6$ & 0.05 & 0.56 \\
Symptomatic localized lesion (Yes/No) & $77 / 19$ & $22 / 78$ & $<0.0001$ & $<0.0001$ \\
Primary site (Lung/Colorectum/Other) & $19 / 21 / 56$ & $18 / 28 / 54$ & 0.61 & - \\
\hline
\end{tabular}

et al. also performed a population-based palliative radiotherapy survey in the Netherlands [13]. Questionnaires were sent to 1100 family physicians and 498 returned questionnaires were evaluated in the study. Almost all general practitioners who completed the survey $(96 \%)$ considered themselves to be the most important caregiver of terminal patients who received palliation. About $40 \%$ of the respondents assessed their own knowledge of RT as modest, including their knowledge of the application and benefit of a short series of palliative RT. Most responding physicians considered RT effective for painful bone metastases, spinal cord compression, painful local disease, brain metastases and airway obstruction, while the potential effectiveness for managing hemoptysis and hematuria was not as well recognized. In the study, the most important negative factors affecting referrals for 
palliative RT were patient-related ones such as general condition, presumed discomfort and patient preferences; however, the authors discussed how such factors may have been due to family physicians not having sufficient knowledge of palliative RT to relieve symptoms, and concluded that this may have led to fewer referrals for palliative RT.

The present study demonstrated that CT findings alone may provide only a certain amount of information about the indication for RT to non-radiation oncologists. We found that when a CT evaluation was performed for patients with metastatic/recurrent/inoperable cancer, about half may have had an indication for RT without preconditions; $\leq 4$ lesions and/or the existence of a symptomatic, localized lesion strongly suggested an indication for RT. In regards to these two factors, with about half of patients with $\leq 4$ lesions, and $80 \%$ of patients with a symptomatic, localized lesion showing an indication for RT, these patients could almost have been evaluated by additional CT only. This result may increase the number of appropriate referrals for radiotherapy, especially in a palliative setting.

The notion of oligometastases and oligo-recurrences has recently been proposed [14]-[17], with the suggestion that local therapy such as surgery, RT, and radiofrequency ablation to a small number (commonly less than three to four lesions) of gross metastatic sites and recurrences may result in prolonged survival or even a cure [18]. Oligometastases is the state in which the patient shows distant relapses in only a limited number of regions; a controlled primary lesion is not required. The most favorable prognostic factor for oligometastases is the state of the primary lesion, which means that oligometastatic patients with controlled primary lesions achieve significantly better survival than those with active primary lesions [19] [20]. Oligo-recurrence, which is similar to oligometastases, is the state where cancer patients with one to several metastases or recurrences have controlled primary lesions. The biggest difference between oligometastases and oligo-recurrence is in the uncontrolled or controlled primary lesion. In this study, the number of cases with an indication for radical or semi-radical RT, who could potentially be cured or controlled in the long-term, was 33 out of all 96 cases (34\%) that had an indication for some sort of RT. Sixteen of the 33 cases were treated with chemotherapy $(n=5)$ or supportive care $(n$ $=11$ ), so intensive local therapy could not be applied for oligometastatic/oligo-recurrent cases (number of lesion $\leq 4)$. Our result also implied that these curable or, in the long-term, controllable cases may not have had appropriate RT because of a lack of their physician's understanding of RT.

We also often encounter cancer patients with symptomatic localized tumors with, for example, painful bone metastases, airway obstruction, painful local disease, brain metastases, spinal cord compression, hemoptysis, and hematuria, among other symptoms. In the present study, 77 (78\%) of 99 patients with a symptomatic, localized tumor exhibited an indication for RT, but only $35(35 / 77,45 \%)$ of these were actually treated with RT (Figure 2). Our view is that all cancer cases, including cases of metastatic cancer and those after initial therapy, which are in line to receive treatment, should be discussed. However, in fact, most cases for which standard initial treatment is applied are excluded from cases to be discussed because of limitations in time and manpower. In such situations, we could neither intervene in treatment decisions, nor discuss any indications for RT without consulting with physicians or surgeons. We also consider that intervention by radiation oncologists early in the "second or after" treatment decision process to be important because we often observed patients who missed the timing of RT because of a delay in consultation. Our findings could solve this treatment delay to some extent since we found a significant correlation between $\leq 4$ lesions and the existence of a symptomatic, localized lesion, with indications for RT.

Regarding limitations associated with this study. Firstly, this study was a retrospective observational study at a single Japanese institution, which suggests the potential for treatment and/or patient selection bias. For instance, we could not evaluate patients without CT images. Regarding RT indications, these were not always determined solely by a radiation oncologist: refusals by patients and the avoidance of RT by the attending physician need to also be considered.

Secondly, indications for RT were determined by a single radiation oncologist. The oncologist diagnosed patients with metastatic/recurrent/inoperable cancer with reference to their electronic medical records, and determined any indication for RT. This raises the possibility of subjective decision-making having occurred; however, in this study, the rates determined of cancer patients who could benefit from RT were found to be comparable to previous studies [4] [6] [8] [21]. In spite of the demonstrated usefulness of a radiation oncologist in determining indications for RT, an existing shortage of radiation oncologists is a great problem in Japan. A national structure survey of radiation oncology in 2009 revealed that about 700 hospitals possessed linear accelerators, but the workforce of radiation oncologists was approximately 900 full-time equivalents. This has led to the all too typi- 
cal and far from ideal situation of each hospital in Japan having access to the services of a single radiation oncologist.

\section{Conclusion}

In summary, in the current study, screening $\mathrm{CT}$ images with reference to the electronic medical records of patients demonstrated that about half of all patients with metastatic/recurrent/inoperable cancer showed an indication for RT. Moreover, having $\leq 4$ lesions and/or the existence of a symptomatic, localized lesion, strongly suggested an indication for RT. These pivotal findings should benefit radiation oncologists in their determinations of the most appropriate treatments for cancer patients.

\section{Conflict of Interests}

The authors declare that they have no competing interests.

\section{Authors' Contributions}

SY designed the study, collected, analyzed, and interpreted data, and drafted the manuscript. YM, SY, MW, SY, HO, SI, and HS collected and analyzed data. $\mathrm{CH}$, TT, and KS contributed to data analysis. All authors read and approved the final manuscript.

\section{Acknowledgements}

We are greatly and sincerely indebted to all our patients and the physicians at Kitakyushu General Hospital; without their continued support and encouragement, this study would not have been possible. The secretarial assistance of Chizue Hamachi, and work by radiation therapy technologists, Takuji Tanigaki and Koji Shiragami, are sincerely appreciated.

\section{References}

[1] Ashby, M. (1991) The Role of Radiotherapy in Palliative Care. Journal of Pain and Symptom Management, 6, $380-388$. http://dx.doi.org/10.1016/0885-3924(91)90030-8

[2] Coia, L.R., Owen, J.B., Maher, E.J. and Hanks, G.E. (1992) Factors Affecting Treatment Patterns of Radiation Oncology in the United States in the Palliative Treatment of Cancer. Clinical Oncology, 4, 6-10. http://dx.doi.org/10.1016/S0936-6555(05)80762-9

[3] Fine, P. (2002) Palliative Radiation Therapy in End-of-Life Care: Evidence-Based Utilization. American Journal of Hospice \& Palliative Care, 19, 166-170. http://dx.doi.org/10.1177/104990910201900307

[4] Delaney, G., Jacob, S., Featherstone, C. and Barton, M. (2005) The Role of Radiotherapy in Cancer Treatment: Estimating Optimal Utilization from a Review of Evidence-Based Clinical Guidelines. Cancer, 15, 1129-1137. http://dx.doi.org/10.1002/cncr.21324

[5] Tisdale, B.A. (1999) When to Consider Radiation Therapy for Your Patient. American Family Physician, 59, 11771184.

[6] Levitt, S.H. and Leer, J.W. (1996) The Role of Radiotherapy in Sweden: A Landmark Study by the Swedish Council on Technology Assessment in Health Care. Acta Oncologica, 35, 965-966. http://dx.doi.org/10.3109/02841869609100713

[7] MacKillop, W.J., Groome, P.A., Zhang-Salomons, J., Zhou, Y., Feldman-Stewart, D., Paszat, L., et al. (1997) Does a Centralized Radiotherapy System Provide Adequate Access to Care? Journal of Clinical Oncology, 15, 1261-1271.

[8] ISCRO (1991) Radiation Oncology in Integrated Cancer Management: Report of the Inter-Society Council for Radiation Oncology. American College of Radiology, Reston.

[9] Teshima, T., Numasaki, H., Shibuya, H., Nishio, M., Ikeda, H., Sekiguchi, K., et al. (2010) Japanese Structure Survey of Radiation Oncology in 2007 Based on Institutional Stratification of Patterns of Care Study. International Journal of Radiation Oncology*Biology*Physics, 78, 1483-1493. http://dx.doi.org/10.1016/j.ijrobp.2009.10.019

[10] Eisenhauer, E.A., Therasse, P., Bogaerts, J., Schwartz, L.H., Sargent, D., Ford, R., et al. (2009) New Response Evaluation Criteria in Solid Tumours: Revised RECIST Guideline (Version 1.1). European Journal of Cancer, 45, 228-247. http://dx.doi.org/10.1016/j.ejca.2008.10.026

[11] Therasse, P., Arbuck, S.G., Eisenhauer, E.A., Wanders, J., Kaplan, R.S., Rubinstein, L., et al. (2000) New Guidelines 
to Evaluate the Response to Treatment in Solid Tumors. Journal of the National Cancer Institute, 92, 205-216. http://dx.doi.org/10.1093/jnci/92.3.205

[12] Samant, R.S., Fitzgibbon, E., Meng, J. and Graham, I.D. (2006) Family Physicians' Perspectives Regarding Palliative Radiotherapy. Radiotherapy \& Oncology, 78, 101-106. http://dx.doi.org/10.1016/j.radonc.2005.11.008

[13] Vulto, A., van Bommel, M., Poortmans, P., Lybeert, M., Louwman, M., Baart, R., et al. (2009) General Practitioners and Referral for Palliative Radiotherapy: A Population-Based Survey. Radiotherapy \& Oncology, 91, 267-270.

[14] Niibe, Y., Kenjo, M., Kazumoto, T., Michimoto, K., Takayama, M., Yamauchi, C., et al. (2006) Multi-Institutional Study of Radiation Therapy for Isolated Para-Aortic Lymph Node Recurrence in Uterine Cervical Carcinoma: 84 Subjects of a Population of More than 5000. International Journal of Radiation Oncology*Biology*Physics, 66, 13661369. http://dx.doi.org/10.1016/j.ijrobp.2006.07.1384

[15] Hellman, S. and Weichselbaum, R.R. (1995) Oligometastases. Journal of Clinical Oncology, 13, 8-10.

[16] Niibe, Y., Kuranami, M., Matsunaga, K., Takaya, M., Kakita, S., Hara, T., et al. (2008) Value of High-Dose Radiation Therapy for Isolated Osseous Metastasis in Breast Cancer in Terms of Oligo-Recurrence. Anticancer Research, 28, 3929-3931.

[17] Niibe, Y. and Hayakawa, K. (2010) Oligometastases and Oligorecurrence: The New Era of Cancer Therapy. Japanese Journal of Clinical Oncology, 40, 107-111. http://dx.doi.org/10.1093/jjco/hyp167

[18] Inoue, T., Katoh, N., Aoyama, H., Onimaru, R., Taguchi, H., Onodera, S., et al. (2010) Clinical Outcomes of Stereotactic Brain and/or Body Radiotherapy for Patients with Oligometastatic lesions. Japanese Journal of Clinical Oncology, 40, 788-794. http://dx.doi.org/10.1093/ijco/hyq044

[19] Niibe, Y., Nishimura, T., Inoue, T., Karasawa, K., Shioyama, Y., Ogawa, Y., Takemoto, M. and Shirato, H. (2010) Oligometastases of Brain only in Patients with Non-Small Cell Lung Cancer (NSCLC) Treated with Stereotactic Irradiation (STI): A Multi-Institutional Study. International Journal of Radiation Oncology*Biology*Physics, 78, S497. http://dx.doi.org/10.1016/j.ijrobp.2010.07.1163

[20] Guerra, J.L.L., Gomez, D., Zhuang, Y., Hong, D.S., Heymach, J.V., Swisher, S.G., Lin, S.H., Komaki, R., Cox, J.D. and Liao, Z. (2012) Prognostic Impact of Radiation Therapy to the Primary Tumor in Patients with Non-Small Cell Lung Cancer and Oligometastasis at Diagnosis. International Journal of Radiation Oncology*Biology*Physics, 84, 6167. http://dx.doi.org/10.1016/j.ijrobp.2012.02.054

[21] Mackillop, W.J., Groome, P.A., Zhang-Salomons, J., Zhou, Y., Feldman-Stewart, D., et al. (1997) Does a Centralized Radiotherapy System Provide Adequate Access to Care? Journal of Clinical Oncology, 15, 1261-1271. 